\section{Transverse myelitis following general and thoracic epidural anaesthesia}

\author{
MOJCA DRNOVSEK GLOBOKAR - VESNA PAVER ERZEN • \\ VESNA NOVAK JANKOVIC
}

MOJCA DRNOVSEK GLOBOKAR $(\bowtie) \cdot$ VESNA PAVER ERZEN •

VESNA NOVAK JANKOVIC

Clinical Department of Anesthesiology and Intensive Therapy University Medical Centre Ljubljana Zaloska 7. 1000 Ljubljana, Slovenia Phone: +386 15222234 Fax: +38615222234

E-mail: mojca.drnovsek@kclj.si

\begin{abstract}
Acute bacterial transverse myelitis, secondary to an epidural catheter, developed in a 49-year-old man who underwent surgery for carcinoma of the left lung. Left pneumonectomy was performed under combined general and epidural (Th6-7) anesthesia. The operative procedure, anaesthesia and early postoperative course were uneventful. On the fitth postoperative day, the patient developed neurological deficits consistent with high-level paraplegia. Elevated inflammatory parameters along with the results of cerebrospinal fluid analysis and magnetic resonance imaging were suggestive of acute transverse bacterial myelitis of the dorsal part of the spinal cord secondary to epidural analgesia. Despite prompt antibiotic therapy, anti-oedema treatment and rehabilitation, the neurological deficit failed to resolve.
\end{abstract}

Keywords: epidural anaesthesia, paraplegia, transverse myelitis

\section{Introduction}

Epidural analgesia provides effective relief of acute postoperative pain and reduces postoperative morbidity and mortality. Transverse myelitis is an infrequent complication of central nervous system blockade. Individual cases have been reported in connection with spinal, epidural and even general anaesthesia.

\section{Case report}

A 49-year-old professional driver was scheduled to undergo a left pneumonectomy for planocellular carcinoma of the left main bronchus. Preoperative evaluation revealed that the patient had been treated for hypertension for 15 years and hyperlipidaemia for the previous month. He reported smoking 20 cigarettes per day for 20 years and occasional use of alcohol. There was no history of allergy, and he had no previous surgery. He was obviously overweight $(166 \mathrm{~cm}, 93 \mathrm{~kg})$. Preoperative laboratory findings were within normal limits. Chest radiography revealed decreased ventilation of the entire left lung, absence of clear infiltrates and a mild mediastinal shift to the left. Electrocardiography (EKG) demonstrated sinus rhythm with signs of Wolff-Parkinson-White syndrome (WPW sy) and a normal QRS axis. Respiratory function tests showed the following values: forced vital capacity (FVC) $3880 \mathrm{ml}$ (85\%), forced expiratory volume in 1 s (FEV 1) 3180 (65\%), Tiffeneau index (TI) $78 \%$. Carbon dioxide diffusion was within normal limits.

On the day of surgery, an intravenous line, an arterial cannula for invasive blood pressure monitoring, and a central venous catheter were inserted in the induction room and premedication with midazolam $1 \mathrm{mg}$ i.v. and fentanyl 0.05 mg i.v. was administered. Using a sterile procedure and a loss-of-resistance technique with an 18-gauge Tuohy needle, an epidural catheter was inserted at the Th6-7 level. Due to the patient's adiposity and degenerative changes of the spine, which were diagnosed only after the operation, detection of the epidural space and catheter insertion presented considerable technical difficulties. It was accomplished after five unsuccessful attempts. Through the catheter, $80 \mathrm{mg}$ of lidocaine was administered as a test dose, followed by preemptive analgesia with $4 \mathrm{mg}$ of morphine in $10 \mathrm{ml}$ of saline and after that $25 \mathrm{mg}$ of bupivacaine was administered via epidural catheter.

General anaesthesia was induced by intravenous application of midazolam $2 \mathrm{mg}$, fentanyl $0.1 \mathrm{mg}$, propofol 200 $\mathrm{mg}$ and vecuronium $8 \mathrm{mg}$. It was maintained with intravenous propofol, fentanyl and vecuronium. Ventilation of the right lung with 100\% oxygen was performed with a Draeger Cato ventilator via a right double-lumen tube Portex, $41 \mathrm{Fr}$. The intraoperative course was uneventful.

Postoperatively, the patient was transferred to the intensive care unit of the Department of Thoracic Surgery. Post- 
operative pain was adequately controlled with an epidural mixture of morphine hydrochloride $0.1 \mathrm{mg} / \mathrm{ml}$, bupivacaine $0.5 \mathrm{mg} / \mathrm{ml}$, and clonidine $1.5 \mu \mathrm{g} / \mathrm{ml}$, administered at a rate of $1-2 \mathrm{ml} / \mathrm{h}$. Before scheduled therapeutic procedures of respiratory therapy, analgesia was supplemented with intramuscular diclofenac $75 \mathrm{mg}$. The pain score, assessed on a visual analogue scale (VAS), ranged between 2 and 4 . The epidural catheter was removed on the second postoperative day because of pruritus.

On the fifth postoperative day, the patient developed signs of paralytic ileus, associated with formication and progressive paresis in the lower extremities. He had fever and complained of severe abdominal and lumbar pain. On neurological examination, voluntary movement in the lower extremities was preserved only in the toes, and most reflexes were absent, with the exception of the left patellar and the right Achilles reflex. There was loss of sensation below seventh thoracic vertebra level and incontinence of urine and feces was present. Meningeal signs were absent. Magnetic resonance (MR) imaging of the spine displayed a hyperintense signal in the central part of the spinal cord at the level of the third to fifth thoracic vertebra, suggestive of myelopathy or myelitis, there were no signs of spinal cord compression.

A cerebrospinal fluid (CSF) sample revealed increased protein $9.15 \mathrm{~g} / \mathrm{L}$ ( normal 0.15-0.45 g/L), a normal glucose value $2.5 \mathrm{mmol} / \mathrm{L}$ ( normal 2,5$3,9 \mathrm{mmol} / \mathrm{L})$ and an increased leukocyte count $762 \times 106 / L$ ( normal less than $5 \times 10^{6} / \mathrm{L}$ ), mainly on account of neutrophilic granulocytes $666 \times 106 / \mathrm{L}$, lymphocytes $75 \times 106 / L$, monocytes $21 \times 106 / L$. Inflammatory blood parameters were increased WBC $19.4 \times 10^{9}$ , CRP $264 \mathrm{mg} / \mathrm{L}$. At the same time blood glucose was $6.1 \mathrm{mmol} / \mathrm{l}$ (normal 3.6-6.1 mmol/l). Gram stain and subsequent culture of CSF were negative. A tentative diagnosis of acute bacterial transverse myelitis was made according to laboratory findings and good antibiotic response, despite negative culture of the CSF.

The patient was placed on empirical antibiotic treatment with vancomycin $1 \mathrm{~g} / 12 \mathrm{~h}$ i.v. and ceftazidime $2 \mathrm{~g} / 8 \mathrm{~h}$ i.v. and anti-oedemic therapy with dexamethasone $8 \mathrm{mg} / 8 \mathrm{~h}$ i.v. On this regimen he was transferred to the Department of Infectious Diseases. The inflammatory parameters decreased and the fever subsided. A control CSF sample showed a decrease in protein $2.25 \mathrm{~g} / \mathrm{L}$, leukocyte count $7 \times 10^{6} / \mathrm{L}$ and glucose level to $2.9 \mathrm{mmol} / \mathrm{L}$. Despite intensive physical therapy and electrical stimulation, muscle wasting soon became apparent in the legs and thighs. A follow-up MR scan of the spinal cord displayed hydromyelia. Electromyography demonstrated an upper motor neuron lesion.

The patient was transferred to the Rehabilitation Institute of the Republic of Slovenia, Department for injuries and diseases of the spinal cord, where he remained for 18 weeks. The patient achieved complete independence in all daily activities. At the end of therapy, he could walk with crutches and climb stairs using the handrail. Muscle wasting was present in his left leg and thigh resulting in weakness of the left lower extremity.

\section{Discussion}

Epidural analgesia is an effective method of pain relief following thoracotomy. Major lung resection, in particular pulmonectomy, carries a high risk of respiratory complications. Effective analgesia in the postoperative period is essential to ensure the patient's active cooperation in respiratory therapy. Although the method is invasive and carried out in immediate proximity of the central nervous system, serious complications with permanent neurological squeal are rather uncommon. The incidence in the USA being as low as 1:10,0000. (1) The main causes of neurological complications following neuroaxial blockade are needle trauma and local anaesthetic neurotoxicity. (1) According to Bromage three broad categories of complications may be distinguished following neuraxial anaesthesia:

Post-anaesthetic squeal directly attributable to anaesthetic causes

Sequelae solely attributable to nonanaethetic causes

A group where anaesthesia is an incidental but possibly a contributing factor. (2)

Difficult, traumatic puncture of the epidural space may lead to the development of an extradural haematoma, which can act as a nidus for infection. (3) Infections of the epidural space and spinal cord secondary to epidural anaesthesia occur in $0.4 \%$ and $0.7 \%$ of cases, respectively, and mostly take the form of paraspinal or epidural abscess with spinal cord compression, or inflammation of the meningeal membranes. (2-5) Some studies suggest a disproportionately high association of thoracic epidural catheters with epidural abscesses most probably due to difficult insertion and traumatic punction. (2)

Acute transverse myelitis (ATM) has an incidence of 1-4: 1.000000 people per year, affecting individuals of all ages with no sex or familiar predisposition. (5) One third of patients recover with little or no neurologic sequelae, one third with moderate degree and one third with severe disability. (5) It is characterized by acutely or subacutely developing symptoms of motor, sensory and autonomic nervous dysfunction, with a clearly defined rostral sensory border of dysfunction. A diagnosis of ATM requires confirmation of inflammation within the spinal cord. Spinal MRI and CSF analysis are the diagnostic procedures for ATM.

For ATM diagnosis the following are required:

- Abnormal gadolinum enhancement of the spinal cord at MRI or

- CSF pleocythosis or

- Elevated CSF IgG index

According to an etiologic scheme, ATM could be classified into six groups related to:

1. Multiple sclerosis

2. systemic disease (SLE; Sjorgen, antifosfolipid sy) 
3. delayed radiation myelopathy

4. spinal cord infarction

5. parainfections

6. idiopathic

In parainfectious ATM, IgM serology

is positive or lgG levels are fourfold or greater on two successive tests for a specific infectious agent.

The etiology of acute-onset transverse myelitis remains unknown. In 30-60\% of patients there is an antecedent respiratory, gastrointestinal or systemic illness. Neurological injury may be associated with direct infection or remote infection followed by a systemic response causing neurological damage.

Individual sporadic cases of transverse myelitis have been described following intrathecal pump insertion, spinal, epidural anaesthesia and even after general anaesthesia. (6-9) The cases observed after intrathecal pump insertion were caused by Acinetobacter baumanii. When the condition developed following spinal, epidural or general anaesthesia, the causative agent could not be identified. (6-10)
In our patient, the diagnosis of acute bacterial transverse myelitis of the thoracic spinal cord was made on the basis of sudden neurologic disability, CSF analysis and MRI of the spinal cord in association with systemic signs of inflammation. Neurologic deficit appeared quickly with high paraplegia. CSF analysis showed normal glucose, high leucocyte count and increased protein, which is characteristic for bacterial transverse myelitis. A bacterial pathogen was not identified since the epidural catheter had been removed on account of pruritus three days before the onset of symptoms. Culture of cerebrospinal fluid yielded no organisms. At the time of lumbar puncture, the patient was treated with ciprofloxacin and clindamycin, which might explain why his cerebrospinal fluid was sterile. Subsequently, the antibiotic therapy was changed to ceftazidime and vancomycin, which were selected on a purely empirical basis but proved appropriate, leading to a decrease in inflammatory parameters.
The suitability of anti-oedema therapy in transverse myelitis is a matter of debate. Our patient received dexamethasone $8 \mathrm{mg} / 8 \mathrm{~h}$ i.v. for three weeks. According to the literature, higher doses of methylprednisolone (1 g/day for three days, followed by a gradual decrease) have been used with success in a small number of patients. (6-8) Since acute transverse myelitis may have an autoimmune etiology, immunosuppression seems reasonable.

In conclusion, difficult, traumatic puncture of the epidural space may lead to the development of an extradural haematoma, which can act as a nidus for infection. A good aseptic technique is of paramount importance. This includes using a sterile gown, sterile gloves and sufficiently large sterile drapes, thoroughly cleaning the skin prior to catheter insertion, allowing sufficient time for the disinfectant to exert its antibacterial action. Also during postoperative handling of the epidural catheter, aseptic technique is very important.

\section{REFERENCES}

1.Munnur U, Suresh MS. Backache, headache, and neurologic deficit after regional anesthesia. An Cl North Am 2003;21:1.

2.Dawson SJ. Epidural catheter infections. J Hosp Infect 2001;47:3-8

3.Holt HM, Andersen SS, Andersen O, Gahrn-Hansen B, Siboni K. Infections following epidural catheterisation. J Hosp Infect 1995;30:253-60.

4.Brooks K, Pasero C, Hubbard L, Coghlan RH. The Risk of Infection Associated With Epidural Analgesia. Infect Control Hosp Epidermiol 1995;16:725-6.

5.Ubogu EE, Lindenberg JR, Werz MA. Transverse myelitis associated with Acinetobacter baumanii intrathecal pump catheter-related infection. Reg Anesth Pain Med 2003;28:470-4.

6.Kaur R, Girdhar KK, Banerjee N. Transverse myelitis following spinal anaesthesia-a case report. Indian J Anaest 2002;46(5):476-7.

7.Martinez-Garcia E, Pelaez E, Roman JC, Perez-Gallardo A. Transverse myelitis following general and epidural anaesthesia in a paediatric patient. Anaesthesia 2005;60:921-3.

8.Gutowski NJ, Davies AO. Tranverse myelitis following general anesthesia. Anaesthesia 1993;48:44-5.

9.Watanakunakorn C. E. colli meningitis and septicemia associated with an epidural catheter. Clin Infect Dis 1995;21(3):713-4.

10.Berman M, Feldman S, Alter M, Zilber N, Kahane F. Acute transverse myelitis: incidence and etiologic considerations. Neurology $1981 ; 31: 966-71$. 